\title{
Transition between Secondary and University Mathematics
}

\author{
Matiki Talwanga
}

mtalwanga@cut.ac.za

\section{Doi:10.5901/mjss.2014.v5n23p2219}

\begin{abstract}
The first year university student taking mathematics as a major experiences a lot of difficulties in the learning of mathematics. Some people say the new comer does not read, does not do assignments, or is simply ill-prepared for academic studies. To the last point, teachers will always oppose saying the learner's preparation for university studies is adequate. The aim of this article is to expose difficulties that freshman at the university mathematics experience and also highlight the reasons behind those difficulties.
\end{abstract}

\section{Self-Efficacy}

First things first! The way people react to a stimulus differs. Before embracing any new course of actions one should check him/herself to find out if he/she has what it takes to cope with the situation ahead. Any athlete refers to self-efficacy when facing a new challenge. The decision to register in university mathematics should be taken after having such an introspection. Many a time people register for a course in the basis of so and so did it. Therefore I will also be able to do it. Sometimes we close up to soon. We might have been turned off by an unpleasant experience in school mathematics. From such encounter one believes mathematics is too hard to be attempted.

This self-assessment leads to establishing the self-efficacy.

Self-efficacy(3) is 'one's self-judgements of personal capabilities to initiate and successfully perform specified tasks at designated levels, expend greater effort and persevere in the face of adversity' (Blake and Lesser, 2006: 655). Mathematics self-efficacy is a person's assessment of their capability to solve problems in Mathematics, and deal with Mathematics related tasks successfully and satisfactorily (Pajares and Miller, 1994).

\section{Didactical Obstacles}

Mathematics is among the worst taught subjects in secondary schools. Mathematics is different from other subjects. The learning and teaching of mathematics is in contrast to subjects such as biology , physics and history where the concept being studied depends on the assertions of some biologist, some physicists or historians. In mathematics there is nothing between the concept and the learner. He/she can prove the assertion by him/herself. The experiment in most cases requires no fancy equipment. A die, a coin, a paper and a pencil might be just enough.

The mathematics in secondary school is presented more or less as a set of procedural courses and a combination of visual representation with the emphasis on teaching or learning techniques of manipulating numbers and equations and formulae. When solving the quadratic equation $a x^{2}+b x+c=0$, we use the formula $\Delta=b^{2}-4 a c$. Do we as teachers care showing learners the origin of this formula? Does the teacher mind showing why for instance the product of two negative numbers is positive. It is a fact that for learners and the general public mathematics is all about numbers and formulae. This always results with learners asking the what do we use this for? question. The notion of limit of functions, convergence of sequences and series for example are taught to learners with emphasis on finding the limit of the function, etc... Little or nothing is said about proving the existence or showing that "this" is the limit of "that".

Most topics in university mathematics are conceptual in nature. Here students are required to prove the existence say of some particular element; or are asked to come with a counter example, etc... Students have to navigate from the culture of "hands-off", where the teacher does most work to that of "hands-on", where they themselves must actively participate in their learning. For that reason, textbooks should be written in a way that students are encouraged to interact with the text. Questions in the form of (Why?), which might encourage the reader to pause and think about the current discussion should be found in various places throughout the text. Another aspect which might motivate a student to an interest in mathematics is the insertion in the text of various cases of the application of the mathematical concepts being 
studied in areas of public interest such as agriculture, biology, business, economics, medicine, physics or engineering.

As an examination moderator of two mathematics subjects the marked student scripts are sent to me together with the memorandum used by the assessors. I have come to realize that the majority of students would solve an exam question in a particular way, while students of the other group would solve a similar question in their particular way, sometimes different from the method suggested by the assessors in the memorandum and the methods I would have solved. This leads us into saying that the way mathematics is taught certainly influences how well students learn and respond to it. Difficulties experienced by learners may be due to the method, the approach used to tackle the topic. The marks the students get do not measure the students but rather indicates how well the students performed under a certain mode of instruction. A thorough analysis and search for reason of students' failure to understand mathematics, which leads to a new manner of presenting the same topic in class may yield good results.

Think of proving a set is a group or a lattice and finding equivalence classes ,etc..

\section{Communication Ruptures between Secondary School and University}

There seems to be a lack of communication between the secondary school and the university. In the best interest of learners, teachers and university lecturers, there should be an ongoing dialogue. This dialogue will have many interesting outcomes. Among them this paper will list the most noticeable.

- The dissemination of knowledge. New results can easily be transferred to teachers.

- Teachers on both sides will have the opportunity to know what is going on upstream and downstream, and bring corrective changes to the system. Without such communications how would one know in what manner research reaches teachers in one hand; or whether teachers find the research relevant on the other hand. The lack of communication is therefore not productive.

- Avoid misalignment of programs. Mathematics has a cumulative nature. Topics in the syllabus must be arranged and taught in such a way that one concept learned now prepares the teaching and understanding of others forthcoming ones. Teaching of units in secondary school should be done with the mind of what is to come at the university. Many secondary education systems are tailored with the university models in mind. Maths-Physics option prepares mainly students to take mathematics or physics at the college/university. Biology-chemistry school encourages learners to go for biology, chemistry, agronomy and medicine. If one link is missing, it makes it difficult to build new knowledge upon shacking foundation. This may prompt lecturers to add to their program topics they deem important which their students failed to have in secondary school.

- University researchers will be able to design various assessment tools, different teaching styles and highlight the emphasis of mathematics courses. The culture of problem solving, rigor and proof, application which leads to conceptualization will be established. Because of a lot of administrative tasks in their work load, teachers do not have the luxury of varying assessment methods and other types of teaching materials.

- Teacher professional development will resolve the problem of lack of specialists among teachers, especially in the rural areas.

\section{Diversity, Types and Language of the University Mathematics Courses}

Compared to the three usual courses (algebra, trigonometry geometry) that learners are familiar in secondary school mathematics, the number of new mathematics disciplines which characterize the undergraduate mathematics is terrorizing. To this are added the tales surrounding some lecturers. Communities are always filled with hearsay about a certain lecturer's strictness, rigor or the course past failure rate. The new-comers get to meet their lecturer having some pre-conceived idea about him/her.

The change in the types of mathematics combined with the number of new units make life difficult for the incoming students. They are faced with first time courses such as abstract algebra, linear algebra, topology, real analysis, etc... Each of these disciplines has its own form of proofs and language.

Let take this as example: $\forall \epsilon>0 \exists \mu,\{x \in A ; x=2 n\} . A=B \leftrightarrow A \subset B$ and $B \subset A$ These are new concepts in a language that is unfamiliar to first year students. Students must get used to and make them part of their mathematics vocabulary.

The language of mathematics does not affect students alone. The challenge to mathematicians is to explain, write in such a way that the reader of the text would be able to understand. Many of the articles I have written have been returned with the observation" ill motivated." When one manages to write or explain well to others, he/she does it to 
him/herself as well. To the language issue, we should not forget the accent associated to it.

In most cases because of the universal nature of the university, lecturers in the mathematics department may come from different countries. Even if these people would express themselves adequately in English, one's accent also poses a challenge. They would have accent which are different from the local one. Incoming students may be exposed to this for their first time, but are forced to work out ways of coping with the situation. For example: At Fort Hare university, there were lecturers from RSA, Zimbabwe, DRC, China, Nigeria, India, . At Walter Sisulu, lecturers are from India, Zimbabwe, Zambia, DRC, RSA. This distribution may be similar to others universities as well.

Students should be determined to learn the language of mathematics by long and painful experience of writing, reading mathematics texts and being supervised.

\section{Abstraction of the University Course}

Learners are used to texts referring to someone doing an action, eg. Peter invests a sum of " $x$ ". The university textbook use a more imperative forms such as: "Let $x$ be, consider $x$, assume $y$ ", and sometimes we read statements such as How do we do, how does one solve this? Learners have the tendency of excluding themselves from the we. University mathematics deals more with concepts which do not apply to the natural environment of the student.

Consider the concepts such as group, field, ring and topological space. Such do not relate to anything known by the learner so far. Any attempt to compare these models to the natural or physical object is futile.

\section{Adjustment Time}

Consider a person who moves to a new suburb: They must adjust themselves with the new environment, eg finding the nearest grocery shop, locating the gas station or the medical clinic . In this case of freshman in university mathematics, there is more than one world the student is moving to and must adjust timeously to for him/her to be active. Beside getting used to the new town the university might be located at, the university environment is quite different from that of a high school which is characterized by parental assistance. The university is the place of discovered freedom. In this new set -up one needs to exercise self- discipline. The mathematical society the student is moving to and the language of mathematics are two different worlds. Here you think mathematically and speak mathematically.

The time frame after enrolment at the university counts. I have realized in my mathematics class over the years that the students fail badly the first test of the year. This test is written during the first week of March, which is about one month after the university opening. The results tend to improve after their second test at the end of April.

\section{Conflict in the Mind Concerning Past known Facts}

In most cases students in mathematics want a new concept to first make sense in the context of known facts of the past, which Tall David calls the " met-befores" (1). This effort takes valuable time that could have been used to assimilate the concept. Later the students realize that a new concept is understood in the context of its definition according to the language of its definition. It becomes more clear as one encounters the term more often, its mysteries fading away. Think of the first time we solved this kind of exercise:

$3+4=7$, this is consistent with the past

$3-2=1$ which can read as take away 2 from 3 , we are let 1 which is little.

3).

But $-3-(-2)=-1$. If we read as above we would say $(-2)$ taken away from $(-3)$, we have $(-1)$ which is larger than $(-$

If one is used to the construct that taking away reduces, then there is a conflict when he encounters the last where taking away has increased what we had. Here is another example of conflict.

The set of counting numbers is infinite and it is made of even numbers and odd numbers, which are infinite as well. If we remove all odd numbers from the counting numbers, the remaining set is still infinite. This creates an inconsistent conflict in the mind of a learner because an infinite number subtracted from an infinite number does not make sense that there is still infinite number remaining.

With regards to the met-before, there is always conflict between what the lecturer assumes to be the students' metbefore and the actual student's met-before. There is a discrepancy between what the syllabus prescribes and the depth at which the teacher actually taught. New subjects should be taught with much caution to this fact. 


\section{Under-Preparedness of Students Entering University}

Under-preparedness signals a fact which seriously impedes learning mathematics at university. Anyone intending to study mathematics should be prepared to work hard and aim higher. This self-discipline seems not to have been taken in consideration when the pass mark in secondary was determined by authorities. A pass mark under $50 \%$ does not call for extra effort. Let alone the $34 \%$ and $40 \%$ as minimum pass requirement. Where will a student get that extra activity if he/she has come to the university without that culture of hard work? The under-preparedness is due to the lack of units in the secondary curriculum which link closely with those in first year university mathematics.

We can check that little is said about logics, sets theory, abstract and linear algebra, affine geometry in secondary school. These courses are the bulk of first year mathematics. Lecturers do not have extra time at their disposal to go over to the details of a prerequisite. By its nature the university brings together students with different back grounds, countries or educational systems. Some would have done this course, some another course. In my mathematics class of calculus to the first year mechanical, civil and electrical engineering, I had students who had come from the Democratic Republic of the Congo (DRC) and Zimbabwe who claimed to have done "Integration" in secondary school. The majority of my students were matriculants from RSA, who were being taught this concept for the first time.

\section{How to Read a Mathematics Text Book?}

Many people have different ways of reading a newspaper. Some read it backward, starting with the sports and weather sections and finally the front section. In reading the paper this way, these people argue that important news tends to be dull and are as such put in the back pages. The language used in newspapers is redundant. A word can be deleted in a sentence but yet the remaining text is still understandable. Consider the sentence, The sky was darkened by heavy clouds. Suddenly the ... started pouring. The absence of the word rain does not affect the statement.

Consider the following sentence. In the space provided, write all the ....multiples of 4 and 6 ..... than 30.

The omission of the word "common" before the word multiple and the omission of the words "less than" would render the task impossible for the learners.

A mathematics textbook is to be read in such a way that the reader becomes an active participant, practically a cowriter of the book. In the sense that the reader must stop at various steps to check the calculations, draw a missing diagram, give some example to try illustrate claims made by the author. Each detail has its meaning. One should concentrate on details, reading each word, each symbol, checking each statement. In summarizing this Sherman K. Stein (4) says mathematics is a verb, not just a noun. There are often several different forms in which to express a numerical or algebraic expression. The answer the reader finds may look different at a glance from the one suggested by the author. One should not throw the towel immediately. A few transformations may reveal later that the same result was obtained.

$$
\text { Ex: }\left(\frac{1}{\sin x}\right)^{2} \text { can just be expressed as } 1+(\cot x)^{2} ; \frac{1}{1+\sqrt{2}} \text { as }-(1-\sqrt{2}) \text {. }
$$

\section{The Supplementary Instruction (S.I)}

I applaud in strong terms the idea of supplementary instruction being implemented in all mathematics subjects at undergraduate level. The supplementary instructor (S.I) is a strong student in one subject, chosen by the lecturer to facilitate some learning activities in the previous class. A second year student may be chosen to help students of the first year. The S.I receives an incentive. The most important aspect is, as I have realized, that these activities boost their confidence and the mastery of the subject is apparent. Two of the S.I's attached to the subjects I teach (ITW10AB and $B C L 11 A B)$ have improved in the subject now compared to the status they were in the previous year. I marvel at them when we discuss the activity they prepare, prior to their going to meet students. This reminds me of the Latin motto "docendo discimus", which means we learn while we teach. In this regard, I must confess that in my class, when marking a class test, I would pick first the best student's script. The way this student would answer a question and the marks obtained give me a clue of how the class understood the topic. Sometime I would discover my own mistake in the memorandum.

\section{Assumption Factor}

When driving my car on the high way, I always assume that the driver in the next lane is experienced, he/she has a driver licence, he/she will indicate when he wants to change lane, and his/her car is in good shape. I also assume that the car 
brakes are going to bring the car to a halt at my command.

This type of assumption has unfortunately been taken to the world of mathematics. Unless one is told, we never assume that these lines are parallel. We should assume that the drawing is done at scale and consider that segments have the same length.

When we say that $(x y>0)$ if both $x$ and $y$ have the same signs. We are not assuming. This is a fact. But when we have $x-y=2$; we do not assume that $\mathrm{x}$ is necessarily a positive number. Consider the set $\mathrm{H}$ of husbands and the set $\mathrm{W}$ of their wives. We can make the assumption that these two sets have the same number of elements. The assumption here is that there is no bigamy.

Consider the sets of numbers $[0,3]$ and $[0,1]$;

One may wrongly say that $[0,3]$ has three times the number of elements of $[0,1]$. The assumption being that the length of the first segment is three times that of the second segment. As parts of the set of reals numbers, the two intervals have same number of elements. Having the same number of elements has nothing to do with length of segments. Another example comes to my mind. The stretch of road from Alice to East London in Eastern Cape is 120 $\mathrm{km}$. So also is the distance from East London to Butterworth. There are a number of towns between Alice and East London. But there is no town between East London and Butterworth. The length of the segments of the road does not influence the number of towns.

The difficulties one experiences in mathematics might be due to this or that factor. One fact remains. No university study is too easy for anyone. Hard work is required of everyone resulting in the mastery of the subject.

\section{References}

The transition to formal thinking in mathematics (David Tall) 2008,Mathematics education research journal. Difficulties in the passage from secondary to tertiary education (Guzman M, Hodgson B, Robert A Vrillani, 1998 Mwamwenda, Tuntufye S, mathematics self-efficacy,2009, The journal of independent Teaching and Learning, vol 4. Sherman K. Stein, Strength in numbers, 1996, John Wiley \& Sons, Inc. 